\title{
An Intelligent Policy-based networking environment for dynamic negotiation, provisioning and control of QoS
}

\author{
Francine Krief, Zeina Jrad \\ LIPN Laboratory, University of Paris XIII \\ Avenue Jean-Baptiste Clément 99, 93430 Villetaneuse, France. \\ \{krief,zj\}@lipn.univ-paris13.fr
}

\begin{abstract}
The development of real time applications (voice, video) implies the introduction of QoS mechanisms into IP networks. The IETF proposed a general framework for the control and management of these IP networks. Configuration, control and management are governed by policy rules. To provide a service with QoS guaranties, it is interesting dynamically to be able to create or modify these policy rules. In this paper we investigates the use of intelligent agents for dynamic negotiation, provisioning and control of QoS in policy-based networking environment.
\end{abstract}

Key words: QoS, policy-based management, intelligent agents, multi-agent system, SLA, SLS, COPS, COPS-SLS

\section{INTRODUCTION}

Internet is based on simple principles, providing only one "best-effort" service without any guarantees. However, with the considerable development of the Internet and multimedia applications, a best-effort service is no more sufficient. To provide QoS in best-effort IP networks, the IETF developed the IntServ and DiffServ architectures. The implementation of QoS mechanisms is a very heavy task because of the abundance of QoS information and the dynamic nature of QoS configurations. The operator must control the attribution of network resources according to the

The original version of this chapter was revised: The copyright line was incorrect. This has been corrected. The Erratum to this chapter is available at DOI: 10.1007/978-0-387-35620-4_43 
applications and users characteristics. In order to simplify and to be able to automate the router configurations, the IETF proposed a general framework called policy-based networking [1] for the control and management of these IP networks. But the high level of management complexity of theses networks makes difficult any dynamism. We propose, in this paper, to introduce some intelligence in the policy-based management to increase flexibility and we retained, for this purpose, multi-agent systems (MAS) for their distributed nature and their adaptability. Then we propose an intelligent architecture for the dynamic negotiation, provisioning and control of QoS in a policy-based networking environment. Finally we present future works.

\section{POLICY-BASED NETWORKING AND MULTI- AGENT SYSTEM}

The necessary functional entities of the policy-based management framework are the Policy Manager, the Policy Enforcement Point (PEP), the policy Decision Point (PDP) and the Policy Repository [2]. COPS (Common Open Policy Service) [3] is a communication protocol between the PEP and the PDP and several extensions have been proposed [4, 5] such as COPSSLS for SLS negotiation between a client and an ISP [6]. To have a guaranty of service level, a user signs a Service Level Agreement (SLA) with its service provider. To define the services applied to a data flow, technical parameters and values are associated with the SLA and are called SLS (Service Level Specifications). Policy-based management is dynamic. If policies rules change, the network behavior changes. The CADENUS project [7] proposes an integrated solution for the dynamic creation, configuration and provisioning of end-user services with QoS guaranties in premium IP network. The distributed and dynamic environment in which operators are supposed to work are what make us choose a MAS (known for its adaptability) to represent the process of negotiation, provisioning and control of QoS. A MAS is an organised set of software or physical entities responsible for the accomplishment of certain tasks in an autonomous way [8]. The following section describes the proposed architecture.

\section{INTELLIGENT ARCHITECTURE FOR DYNAMIC QOS NEGOTIATION, PROVISIONING AND CONTROL}

The introduction of intelligent agents in network environments is an important research topic. This trend is motivated by the desire to use agents 
in order to help the user (e.g. assistant agent), to perform complicated tasks (e.g. resources allocation) and to automate some processes (e.g. control). Figure 1 represents the proposed intelligent architecture. This architecture is in conformity with Policy-based management and CADENUS architecture presented in section 2 .

\subsection{Dynamic negotiation of QoS and dynamic selection of service provider}

We propose a system that plays the role of Intelligent Interface between

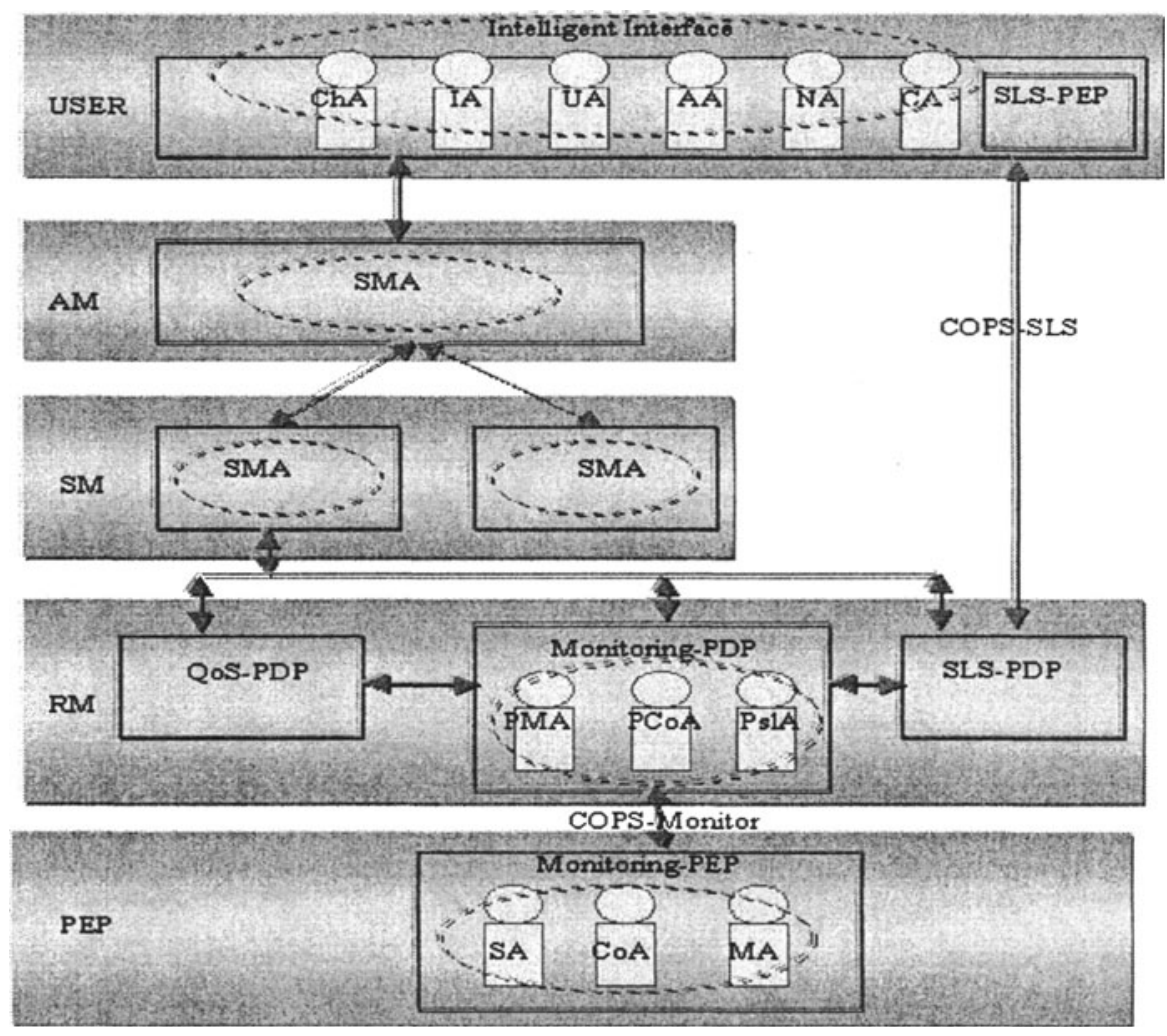

Figure 1. Global architecture

the user and the network. This interface is to be installed on the terminal and has to accomplish two tasks which are the dynamic negotiation of the Service Level Specifications and the selection of the service provider who best answers the demands of the user. The realisation of the two tasks presented above requires the presence of a MAS in the intelligent interface. The communication, the co-ordination and the negotiation among the agents lead the system to a more efficient output. Each agent has a base of 
knowledge in which he can save information and from which he can retrieve data that helps him taking his decisions. The agents in the architecture can be described as follows:

\subsubsection{User Agent (UA)}

The first step consists of the identification of the user who tries to login on the terminal. Once the agent gets the name and the password of the user, he will search in his base of knowledge trying to find the query that corresponds to that person and that contains information about the previous work of the user. This data is what constitutes the profile of that user. Then, UA keeps analysing his behaviour and modifies the profile accordingly. For new users, UA collects necessary information from their works.

\subsubsection{Interface Agent (IA)}

It determines the contents of the graphical messages that the system needs to send to the user in order to inform him or to get information from him. In the case of an old user, IA can use messages that has been already communicated to the user since it knows already his previous choices and needs.

\subsubsection{Choice Agent (ChA)}

According to the profile of the user, this agent will select the Access Mediator that leads to the service provider who best answers the requirements of this profile. The Access Mediator may transmit to ChA possible new offers presented by the service providers with whom it is connected (variation of QoS prices, free services, etc.). On the other side, for more efficiency, we propose the integration of a MAS plate-form in the Access Mediator since it participates in the choice of the service provider and to use FIPA [9] as a communication protocol.

\subsubsection{Application Agent (AA)}

This agent determines the profile of the active application. The same application used by two different users will not inevitably claim the same performance guarantees. This depends on the user's profile and his constraints on time, budget or other choices. AA should be able to associate a profile to the actual application depending on its type and on the user's profile.

\subsubsection{Negotiation Agent (NA)}

This agent takes the application's profile and the user's profile and associates to them the appropriate SLS parameters. NA is an intelligent agent that should learn how to determine the values of the SLS parameters 
according to the requirements of the user and the application. Once the values of the SLS parameters are determined, they should be submitted to the SLS-PEP of the terminal to negotiate for the QoS with the SLS-PDP of the service provider.

\subsubsection{Control Agent (CA)}

This agent plays the role of monitoring in order to verify that the process of negotiation is respected by both of the two ends. CA will also respond to the demand of the service provider to re-negotiate the QoS in case he can not preserve what he accepted to do. He will also verify that the user pays effectively the services that he got and that there is no cheating in the calculated fees.

\subsection{Policies validation before provisioning}

Policies are set of rules to administer, manage and control access to network resources. IETF has specified the information model to be used to represent political information. The introduction of new policies in the network may lead to a conflict with other existing policies. Therefore, we propose to introduce intelligent agents in the Service Mediator to detect possible conflict before a new policy is implemented in the real network environment. The Service Mediator is in charge of dispatching the policies toward the different Resource Mediators. In the QoS domain we have identified three Resource Mediators: the QoS-PDP responsible for implementing QoS policies in the network, the SLS-PDP for the SLS negotiation with the SLS-PEP and the Monitoring-PDP for the QoS control. Moreover, a new policy has to be validated before being used, that's why the MAS must also verify properties and possible inconsistencies of a new policy. The policies which are not validated are returned to the Access Mediator. We are working on the architecture of this SMA with ALCATEL (MAGDA2 project) [10].

\subsection{Dynamic control of QoS}

PEP can also perform an active role to collect the necessary information that represents current network or traffic situation. Thus, we propose [12] to delegate the QoS monitoring to the different PEPs and we introduce intelligent agents in PEP whose function is to detect possible SLA violations. The PEP contains three types of agents. The SondeAgent (SA) that collects indicators of QoS and transmits them to MA. The MonitoringAgent (MA) that compares the data collected with those sent by the PslA. If it detects a risk of SLA violation it transmits them to the 
CopsAgent (CoA) which manages the communication with the PDP via COPS. On the other side, the Monitoring-PDP contains three types of agents. The PDPcopsAgent (PCoA) which manages the communication with the PEP via COPS. The PDPMonAgent (PMA) which makes the decision to react by asking for a re-dimensioning of the network or a re-negotiation of the SLS with the user using COPS-SLS. The PDPslsAgent (PslA) which sends the values of the SLS to the MA. Finally, we propose a new extension of COPS to transport QoS control information that we call COPS-monitor.

\section{CONCLUSION AND FUTURE WORK}

In this paper we proposed an intelligent policy-based networking environment for dynamic negotiation, provisioning and control of QoS. The architecture is in conformity with the CADENUS architecture. Future works concern the implementation of the user intelligent interface in the context of ARCADE project [12] and the specification of COPS-Monitor for QoS control. We work also with ALCATEL (MAGDA2 project) on policy validation and detection of SLA violation in a GMPLS environment. We are also interested by the specification of an architecture which facilitates the deployment of intelligent agents in a policy-based networking environment and which contains mobile agents.

\section{BIBLIOGRAPHY}

[1] R. Yavatkar, D. Pendarakis, R. Guerin, "A Framework for Policy Based Admission Control", RFC 2753, January 2000.

[2] J. Tjaja Hokmoro, "SLA Enforced by Policy", thesis, University of Twente, June 2001

[3] J. Boyle, R. Cohen, D. Durham, S. Herzog, R. Raja, A. Sastry, "The COPS (Common Open Policy Service) Protocol", RFC 2748, January 2000.

[4] S . Herzog, J. Boyle, R. Cohen, D. Durham, R. Rajan, A. Sastry, " COPS usage for RSVP", RFC 2749, January 2000.

[5] K. Chan, J. Seligson, D. Durham, S. Gai, K. McCloghrie, S. Herzog, F. Reichmeyer, R. Yavatkar, A. Smith, " COPS Usage for Policy Provisioning", March 2001.

[6] T.M.T. Nguyen, G. Pujolle, N. Boukhatem N, "COPS usage for SLS negotiation", draftnguyen-rap-cops-sls-00.txt, Work in progress, 2001.

[7] CADENUS Project, "QoS Control in SLA Networks", IST-1999-11017, March 2001.

[8] J.-P. Briot, Y. Demazeau, "principles and architecture of MAS", 2002.

[9] http://www.fipa.org/

[10] RNRT MAGDA2 Project, URL : http//www.telecom.gouv.fr/rnrt/

[11] N. Boukhatem, B. Campedel, H. Chaouchi, V. Guyot, F. Krief, T.M.T. Nguyen, G. Pujolle, "A New Intelligent Generation for Internet Networks", SmartNet 2002

[12] RNRT ARCADE Project, URL : http//www.telecom.gouv.fr/rnrt/ 
CONTENT 